\title{
Expression of glucose transporter 1 in adult and developing human peripheral nerve
}

\author{
P. Muona ${ }^{1}$, S. Jaakkola ${ }^{1}$, V. Salonen ${ }^{2}$ and J. Peltonen ${ }^{1}$ \\ Departments of ${ }^{1}$ Medical Biochemistry, and ${ }^{2}$ Internal Mediciné, University of Turku, Turku, Finland
}

\begin{abstract}
Summary. Northern hybridization of total RNA isolated from adult human sciatic nerve demonstrated a readily detectable hybridization signal for glucose transporter 1 (GLUT 1) mRNA. Western blot analysis demonstrated that GLUT 1 proteins extracted from adult human and from mature rat sciatic nerves had different electrophoretical mobilities. The migration positions of human and rat GLUT 1 proteins corresponded to $60-70 \mathrm{kDa}$ and $55-60 \mathrm{kDa}$, respectively, as estimated by markers with known molecular masses. Indirect immunofluorescence staining localized GLUT 1 to the perineurium in the adult human sciatic nerve. Only a few endoneurial capillaries of human adult nerve stained positively for GLUT 1, which was in contrast to rat peripheral nerve where most endoneurial capillaries were positive for GLUT 1. In developing human nerves, the staining pattern for GLUT 1 was markedly different from that of the adult nerves: at 14 weeks, the perineurial cells were en-
\end{abstract}

tirely negative for GLUT 1 . Between 22 and 26 weeks of gestation, the staining reaction for GLUT 1 in the perineurium became markedly more prominent, and by 35 weeks the intense perineurial staining resembled that observed in the adult human nerves. In contrast to adult nerves, both endoand epineurial blood vessels stained intensely for GLUT 1 in the fetal samples. Thus, the immunoreactivity for GLUT 1 in the perineurium seems to increase concomitant with the maturation of barrier properties of perineurium, whereas the transient expression of GLUT 1 in the vasculature of developing nerve may have a specific role in the proliferating endothelial cells.

Key words: Glucose transporter, endothelial cell, bloodnerve barrier, perineurium, peripheral nerve, development.
During the past few years, a family of sodium-independent glucose transporter molecules (GLUTs) responsible for facilitated diffusion of glucose into mammalian cells has been characterized [1,2]. Five isoforms of glucose transporters are known: GLUT 1 [3] and GLUT 3 [4] may be responsible for basal glucose uptake into cells; GLUT2 [5] mediates the bi-directional glucose transport in the hepatocytes, and may have a role in the glucose-sensing system of the pancreatic beta cells; GLUT 4 [6] is responsible for most of the insulin-stimulated glucose uptake in the muscle and fat tissues; and GLUT 5 [7] has a role in the dietary absorption of glucose from the intestine [1]. The expression of GLUTs by various cell types, e.g. brain glial cells and connective tissue cells of peripheral nerve [8,9], is in part regulated by ambient glucose concentration, and may thus undergo changes in diabetes mellitus.

Peripheral nerves are highly dependent on glucose as their major energy source [10]. In mature rat and canine nerves, GLUT 1 epitopes have been localized to the peri- neurium and endothelial cells of the endoneurial capillaries $[9,11,12]$; i.e. structures that are responsible for maintaining the blood-nerve barrier, which prevents free access of molecules from circulation and surrounding tissue into the endoneurial space and thus serves to maintain the endoneurial homeostasis [13].

In previous studies, marked changes of GLUT 1 mRNA expression in developing rat tissues have been observed $[14,15]$. Furthermore, several tissues such as liver and heart muscle that express GLUT 1 only at a very low level in mature animals, show high steady-state levels of GLUT 1 mRNA during the fetal development $[14,15]$. These findings may suggest that GLUT 1 has a specific role during the early development of tissues.

To date, the expression of GLUTs in human peripheral nerve, or during the development of peripheral nerve has not been studied. Thus, our purpose was to investigate the distribution of GLUT 1 both in adult and developing human peripheral nerve. 


\section{Materials and methods}

\section{Tissue samples}

Sciatic nerve samples were obtained from an apparently healthy subject (age, 16 years) who had died in an accident. The time interval between the death and the fixation of the samples was $6 \mathrm{~h}$. A fresh sciatic nerve sample was obtained from a tissue donor (age, 23 years) critically injured in an accident. The nerve sample was fixed immediately after death. The sciatic nerves of four apparently normal human fetuses, 14, 17, 19 and 19 gestational weeks of age, were obtained from the Department of Obstetrics and Gynecology, Turku University Hospital. The nerves were removed within $2 \mathrm{~h}$ after death. The sciatic nerves of four human fetuses, $17,22,26$ and 35 gestational weeks of age, were obtained from the Department of Pathology, University of Turku. In these cases, the nerve samples were obtained within 2 days after death. No abnormalities were found in any of these fetuses on macroscopic or microscopic examination at autopsy. The sciatic nerves were removed under sterile conditions and snap-frozen in liquid nitrogen. Haematoxylin-eosin and toluidine blue stainings of nerve sections were used for routine histology. The study protocol was approved by the Ethical Committee of University of Turku. For control purposes, sciatic nerves were obtained also from an 8-month-old Sprague-Dawley rat. The rat was killed under $\mathrm{CO}_{2}$ anaesthesia, and the sciatic nerves were removed and snap-frozen in liquid nitrogen.

\section{Indirect immunofluorescence staining}

For indirect immunofluorescence staining, snap-frozen samples of sciatic nerves were cut into $5-\mu \mathrm{m}$ sections on silanated glass slides. Prior to staining, the sections were fixed in absolute ethanol at $-20^{\circ} \mathrm{C}$, or alternatively in $100 \%$ acetone at $-20^{\circ} \mathrm{C}, 10 \%$ formaldehyde (diluted with phosphate-buffered saline, $\mathrm{PBS}, \mathrm{pH} 7.4$ ) at room temperature, or $4 \%$ paraformaldehyde (in PBS) at room temperature. The type of fixation did not affect the observed staining pattern for GLUT 1 epitopes. The sections were balanced in PBS with $1 \%$ bovine serum albumin (BSA/PBS), and exposed to polyclonal rabbit antibodies raised against a synthetic peptide that was composed of the 16 COOH-terminal amino acids of human/rat GLUT 1 (courtesy of Dr. B. Thorens, Whitehead Institute, Cambridge, Mass., USA). The antiserum was used in a dilution of 1:500 (in BSA/PBS), and the incubation was carried out overnight at $4^{\circ} \mathrm{C}$. After careful washes in PBS, the samples were incubated with tetramethyl-rhodamine isothiocyanate (TRITC)-conjugated swine immunoglobulins to rabbit IgG (DAKO A/S, Glostrup, Denmark; 1: 100 dilution in BSA/PBS) for $1 \mathrm{~h}$ at room temperature, washed again with PBS, rinsed briefly with distilled water, and mounted. In control reactions, the primary antibody was omitted, replaced with serum from non-immunized rabbits, or preabsorbed with $10 \times$ molar excess of a synthetic peptide similar to that originally used for immunization. The peptide (the 16 $\mathrm{COOH}$-terminal amino acids of human/rat GLUT 1) was synthesized using Applied Biosystems $431 \mathrm{~A}$ automated peptide synthesizer and 9-fluorenylmethoxycarbonyl protection strategy, purified with reversed-phase high-performance liquid chromatography, and analysed by mass spectrometry. The samples were observed and photographed with a Leitz Aristoplan microscope equipped with an epi-illuminator, filters suitable for TRITC-fluorescence, and camera attachment. In all controls, a faint uniform background fluorescence only was observed.

\section{Western blot analysis}

Proteins were extracted from a minced $\sim 0.5 \mathrm{~cm}$ piece of a fascicle of human adult sciatic nerve (obtained from the 23-year-old subject), and from a minced sciatic nerve of an 8-month-old Sprague-Dawley rat, by intermittent vortexing of the sample in a solution containing $4 \%$ sodium dodecyl sulphate (SDS), $0.5 \mathrm{mmol} / \mathrm{l}$ phenylmethylsulphonyl fluoride, $0.1 \mathrm{mmol} / 14$-chloromercuribenzoate, and $5 \mathrm{mmol} / \mathrm{l}$ EDTA [11]. The samples were centrifuged, and the protein contents of the supernatants estimated by the method of Lowry modified by Peterson [16]. Aliquots containing 10-40 $\mu \mathrm{g}$ of the extracted proteins per lane were separated by SDS-PAGE (12\% homogeneous gel), and electrotransferred to nitrocellulose filters (Schleicher \& Schuell, Dassel, FRG). The filters were incubated with the GLUT 1 antiserum (dilution 1:100 in Tris-buffered saline (TBS) $+5 \%$ non-fat dry milk $+0.2 \%$ Nonidet $\mathrm{P}-40(\mathrm{NP}-40)$ ) for 60 min at room temperature [17]. For controls, the antiserum was preabsorbed with $10 \times$ molar excess of a synthetic peptide identical to that used for immunization (see above) prior to incubation of the filters. After washings, the filters were incubated with $8 \times 10^{5} \mathrm{cpm} / \mathrm{ml}$ of ${ }^{125} \mathrm{I}$-labelled protein A (30 $\mathrm{mCi} / \mathrm{mg}$; Amersham International, Amersham, Bucks., UK) in TBS with $5 \%$ non-fat dry milk and $0.2 \% \mathrm{NP}-40$ for $60 \mathrm{~min}$ at room temperature with agitation. Following washes, the filters were exposed to X-ray films (Kodak X-Omat; Eastman Kodak, Rochester, NY, USA) at $-70^{\circ} \mathrm{C}$.

\section{$R N A$ isolation and Northern analyses}

Isolation of total RNA was carried out as described previously [18]. Briefly, the sciatic nerve samples were homogenized in guanidium thiocyanate buffer followed by acid phenol-chloroform extraction, and after centrifugation the RNA was precipitated from the water phase with ethanol. For control purposes, total RNA was extracted also from fetal brain tissue and HT 1080 fibrosarcoma cells. RNA samples $(20 \mu \mathrm{g} /$ lane) were fractionated on $0.9 \%$ agarose gels under denaturing conditions. The RNAs were transferred to nylon filters (Biodyne transfer membrane; Pall Biosupport, East Hills, NY, USA), immobilized by baking at $80^{\circ} \mathrm{C}$, and hybridized with a human GLUT 1 specific cDNA probe (1.6-kilobase (kb) BamHI-PstI fragment of J-12, [3]), which was labelled radioactively by random-priming (Random Primed DNA Labeling Kit; Boehringer-Mannheim, Indianapolis, Ind., USA). The hybridization solution contained $50 \%$ formamide, $50 \mathrm{mmol} / \mathrm{l}$ sodium phosphate ( $\mathrm{pH} 6.5$ ), $0.1 \%$ SDS, $5 \times$ standard saline citrate $(\mathrm{SSC} ; 1 \times \mathrm{SSC}=0.15 \mathrm{~mol} / 1$ sodium chloride and $15 \mathrm{mmol} / \mathrm{l}$ sodium citrate), $5 \times$ Denhardt's solution, and $250 \mu \mathrm{g} / \mathrm{ml}$ calf thymus DNA. After washings (three times for $5 \mathrm{~min}$ in $2 \times \mathrm{SSC}+0.1 \% \mathrm{SDS}$ at room temperature, and two times for $15 \mathrm{~min}$ in $0.1 \times \mathrm{SSC}$ at $60^{\circ} \mathrm{C}$ ) the filters were kept moist and exposed to X-ray films (Kodak X-Omat) at $-70^{\circ} \mathrm{C}$. The probe was removed by incubating the filters in distilled water containing $0.1 \%$ SDS at $90^{\circ} \mathrm{C}$ for $10 \mathrm{~min}$, and the filters were rehybridized with a human GLUT 3 specific cDNA probe $(2.6-\mathrm{kb}$ Sall-Sall insert of pBS-MGT\# 3, [4]).

\section{Results}

\section{Expression of GLUT 1 protein and $m R N A$ in human nerves}

GLUT 1 protein of adult human sciatic nerve was studied by Western blot analysis of proteins extracted from the sciatic nerve of the 23-year-old subject utilizing GLUT 1 specific antiserum and radioactively labelled protein $\mathrm{A}$. Autoradiography revealed an intense double band, the migration position of which corresponded to that of a marker with known molecular mass of 60-70 $\mathrm{kDa}$. The band was completely abolished by preabsorption of the antiserum with a synthetic peptide identical to that originally used for immunization (Fig. 1A). However, GLUT 1 protein of the sciatic nerve of a mature rat was detected as a single band corresponding to $55-60 \mathrm{kDa}$, which is in agreement with previous findings [11]. The band was not detected in the control filter incubated with the preabsorbed antiserum (Fig. 1A). In the human nerve sample, an additional band of high molecular mass was observed 


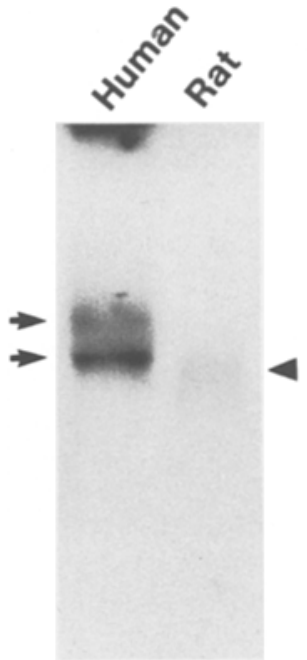

A GLUT 1
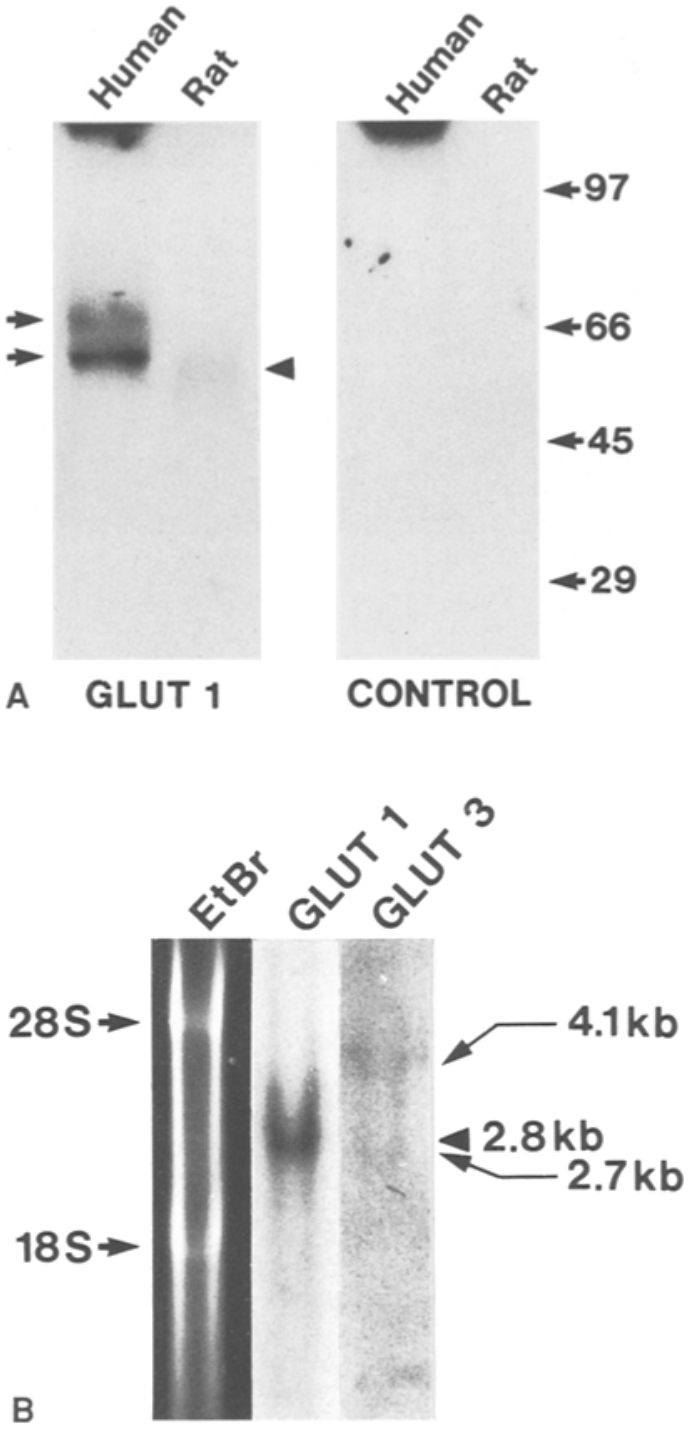

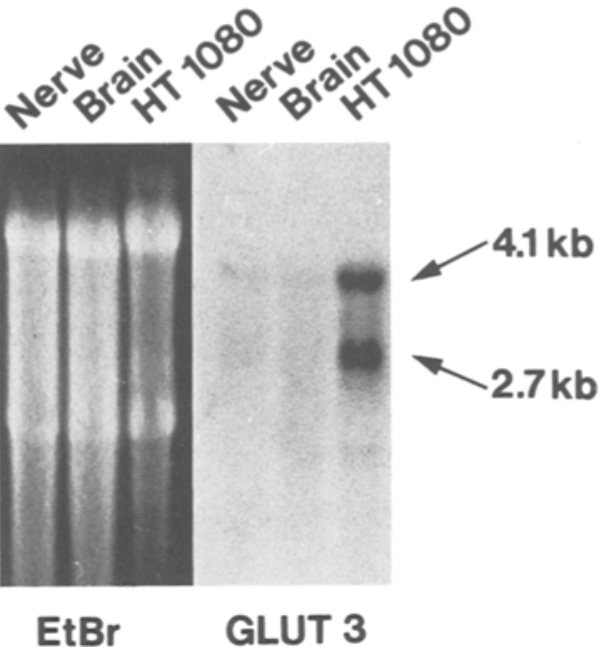

Fig. 1. A Western blot analysis of GLUT 1 protein extracted from adult (23-year-old) human sciatic nerve and from mature (8-month-old) rat sciatic nerve. Samples containing $20 \mu \mathrm{g}$ total protein were subjected to SDS-PAGE, transferred to nitrocellulose filters, and incubated with GLUT 1 specific antiserum followed by incubation with ${ }^{125} \mathrm{I}$-labelled protein $\mathrm{A}$. The mobilities of commercial protein markers with known molecular masses $(\mathrm{kDa})$ are indicated on the right. Note the differences in the mobilities of GLUT 1 proteins extracted from human (arrows on the left) and rat (arrowhead) peripheral nerves. The control filter was incubated with GLUT 1 antiserum that was preabsorbed with a synthetic peptide identical to that originally used for immunization. Only the unspecific band of the human nerve sample is visualized in the control filter

B Northern transfer analysis of GLUT 1 and GLUT $3 \mathrm{mRNAs}$ isolated from adult (23year-old) human sciatic nerve. Total RNA $(20 \mu \mathrm{g})$ was fractionated on $0.9 \%$ agarose gel, transferred to nylon membrane, and hybridized with a cDNA specific for human GLUT 1. Subsequent autoradiography reveals a single 2.8-kb band (arrowhead on the right). After removal of the previous probe (see Materials and methods) the same filter was rehybridized with a cDNA specific for human GLUT 3. Autoradiography reveals two faint bands corresponding to the $4.1-\mathrm{kb}$ and $2.7-\mathrm{kb}$ mRNA transcripts (arrows on the right). Ethidium bromide staining of the same gel is shown on the left (EtBr); migration positions of ribosomal RNAs are indicated (arrows)

C Northern transfer analysis of GLUT 3mRNA extracted from a sciatic nerve of a

19-week-old fetus, from fetal brain and from HT 1080 cells. Autoradiography revealed $4.1-\mathrm{kb}$ and $2.7-\mathrm{kb}$ transcripts (arrows on the right). Ethidium bromide staining is shown on the left (EtBr); migration positions of ribosomal RNAs are indicated by arrows
(Fig.1 A). This band was not abolished by preabsorption of the antibody with the GLUT 1 peptide and was thus considered to be non-specific.

In order to study the expression of GLUT 1 at the mRNA level in the same human nerve as utilized for the Western blot analyses, total RNA was isolated from the nerve and subjected to Northern transfer analyses. Hybridization with a human sequence specific cDNA for GLUT 1 revealed a characteristic $2.8-\mathrm{kb}$ mRNA transcript even after a relatively short $(18 \mathrm{~h})$ exposure (Fig.1B). Rehybridization of the same RNA filters with a human sequence specific cDNA for GLUT 3 resulted in visualization of characteristic $4.1-\mathrm{kb}$ and $2.7-\mathrm{kb}$ transcripts. The bands were, however, barely detectable even after a long (2 weeks) exposure (Fig.1B), indicating a markedly lower level of expression of GLUT 3 in this tissue. To examine whether GLUT 3 mRNA was expressed in fetal peripheral nerve tissue, total RNA was extracted from a sciatic nerve of a 19-week-old fetus and subjected to Northern blot analysis. Typical transcripts of 4.1 and $2.7 \mathrm{~kb}$ were visualized, and the mRNA level roughly corresponded to that observed in fetal brain tissue which was used as a positive control (Fig. 1 C).

\section{Localization of GLUT 1 epitopes in adult sciatic nerves}

Sciatic nerves from two (16- and 23-year-old) subjects showed similar, but not quite identical staining patterns for GLUT 1 epitopes. A positive immunoreaction was associated with the perineurium and some of the vascular components in these mature nerves.

All cell layers of the perineurium showed an intense immunoreaction for GLUT 1 epitopes (Fig.2). Extensions of perineurium formed GLUT 1 positive, one cell layer thick septa which divided the endoneurial space into compartments (Fig. 2A). Furthermore, GLUT 1 positive perineurial cells often extended from the perineurium into the endoneurium to surround a subperineurial blood vessel. Thus, a sheet that was composed of perineurial cells connected the perineurium and the vessel at these sites (Figs. 2B and 3). Similarly, a sheet of perineurium occasionally surrounded an area apparently containing only endoneurial extracellular matrix (Fig.4).

In the adult human sciatic nerves, GLUT 1 positive capillaries were seen in the endoneurium only. Furthermore, compared to mature rat sciatic nerve, only a few endoneurial capillaries were positive for GLUT 1 (Fig.2A, C), 

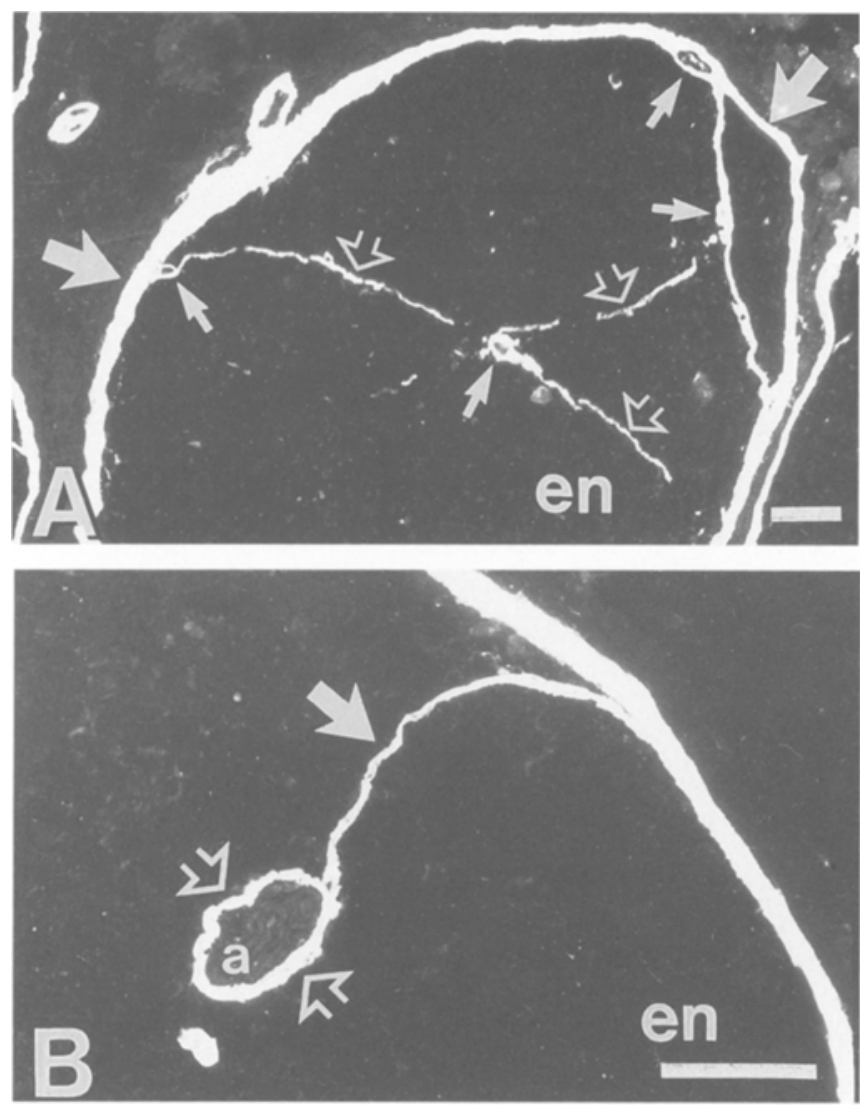

Fig. 2A-D. Indirect immunofluorescence staining for GLUT 1 epitopes in adult (23-year-old) human sciatic nerve (A and $\mathbf{B}$ ) and mature (8-month-old) rat sciatic nerve (C). A Note the intense immunoreaction in association with the perineurium (large arrows). A network of GLUT 1 positive septa (open arrows) divides the human endoneurium into several compartments. The septa are often associated with blood vessels at least at one end (small arrows). B A cellular sheet extends from the perineurium and encircles (open arrows)

even though the total number of capillaries (20-40) per fascicle in these nerves was approximately the same.

GLUT 1 positive endoneurial capillaries were also less frequent in the adult sciatic nerve specimens than in the human fetal nerve samples. In fact, the sample from the 23-year-old subject contained hardly any GLUT 1 positive capillaries (Fig. 2A, B). Larger blood vessels within all connective tissue compartments of the adult nerve were negative for GLUT 1 protein (Fig. 2A, B). However, endoneurial arterioles were often surrounded by a single layer of GLUT 1 positive cells.

\section{GLUT 1 in the perineurium of fetal nerves}

A marked redistribution of GLUT 1 epitopes was observed during the development of the human sciatic nerve between 14 and 35 weeks of gestational age (Fig. 5). Perineurial cells, which showed an intense staining reaction for GLUT 1 in the adult nerve specimens, were apparently negative for GLUT 1 epitopes in the sciatic nerve from the youngest fetus studied (14 weeks) (Fig. 5A). At this age, the perineurium was composed of two to three cell layers around each fascicle as estimated by routine histology.
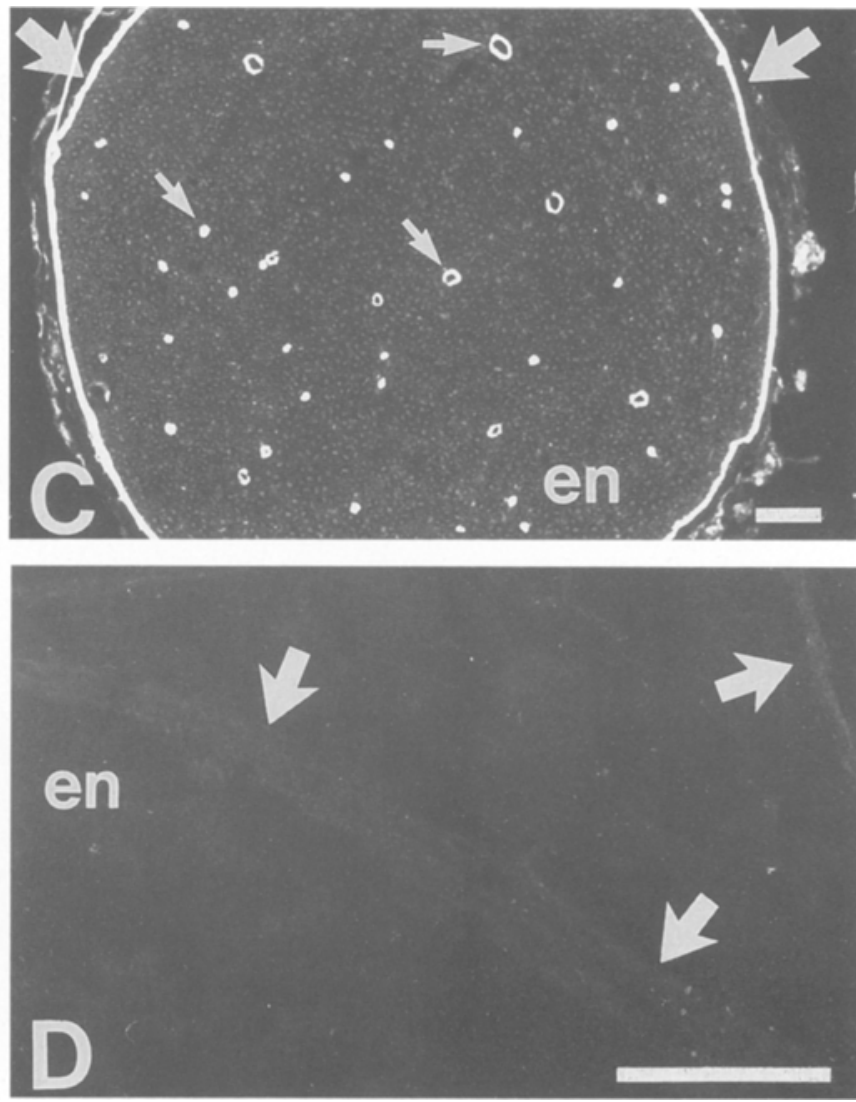

an endoneurial, thick-walled blood vessel, presumably an arteriole (a) in the human nerve. C Perineurium (large arrows) and numerous endoneurial capillaries (small arrows) stain positively for GLUT 1 in a sciatic nerve obtained from a mature (8-month-old) rat. D Control staining of human sciatic nerve with GLUT 1 antibody preabsorbed with a synthetic peptide identical to the antigen originally used for immunization. Perineuria are indicated with arrows. Bar, $100 \mu \mathrm{m}$. en $=$ endoneurium

At 17 weeks of age, occasional short stretches of perineurial cell layers stained positively for GLUT 1 (Fig. 5B). However, most of the apparent perineurial staining was associated with blood vessels around and in the perineurium.

At 19 weeks of age, most of the immunoreaction for GLUT 1 in the perineurium was associated with the perineurial cells (Fig. 5C). Furthermore, perineuria of most fascicles showed a continuous staining for GLUT 1, whereas some fascicles were devoid of perineurial cell-associated staining. In contrast, at 22 weeks all fascicles showed a positive perineurial staining, but the staining pattern was not always continuous (Fig. 5D).

The perineurial staining in the samples from the 26and 35-week-old fetuses was markedly different when compared to the samples obtained from the younger fetuses: the prominent, continuous staining of the perineurial cells at 26 and 35 weeks very much resembled that seen in the adult nerve samples (Fig. 5E, F).

\section{GLUT 1 in the vasculature of fetal nerves}

At 14 weeks of age, a few single GLUT 1 positive cells were seen along the perineurium (Fig. $5 \mathrm{~A}$ ). These cells could be identified neither as perineurial nor as endothe- 


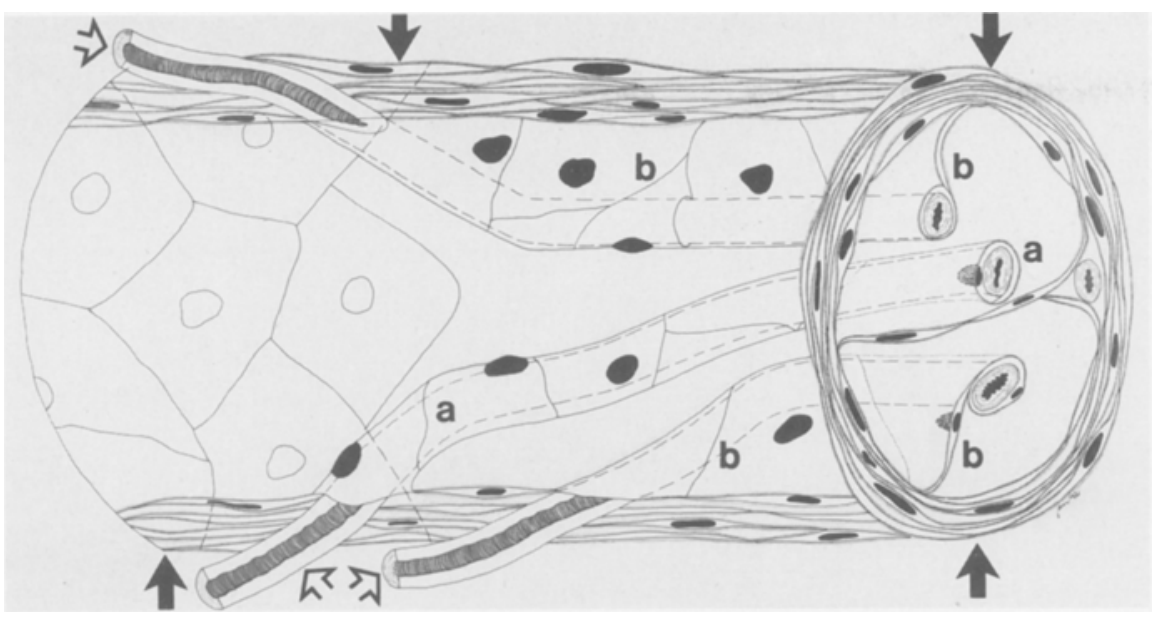

Fig.3. A schematic presentation of GLUT 1 positive, perineurium-derived structures in relation to the endoneurial blood vessels in a fascicle of adult human nerve. Blood vessels entering the endoneurium may be ensheathed by a layer of perineurial cells in two different ways, as deduced from findings shown in Figure 1. a: A layer of perineurial cells may form a tubular sleeve $[12,26]$ around the blood vessel (open arrows) traversing the perineurium (arrows). b: A sheet of perineurial cells may leave the perineurium with the blood vessel, simultaneously investing the blood vessel and connecting it with the perineurium
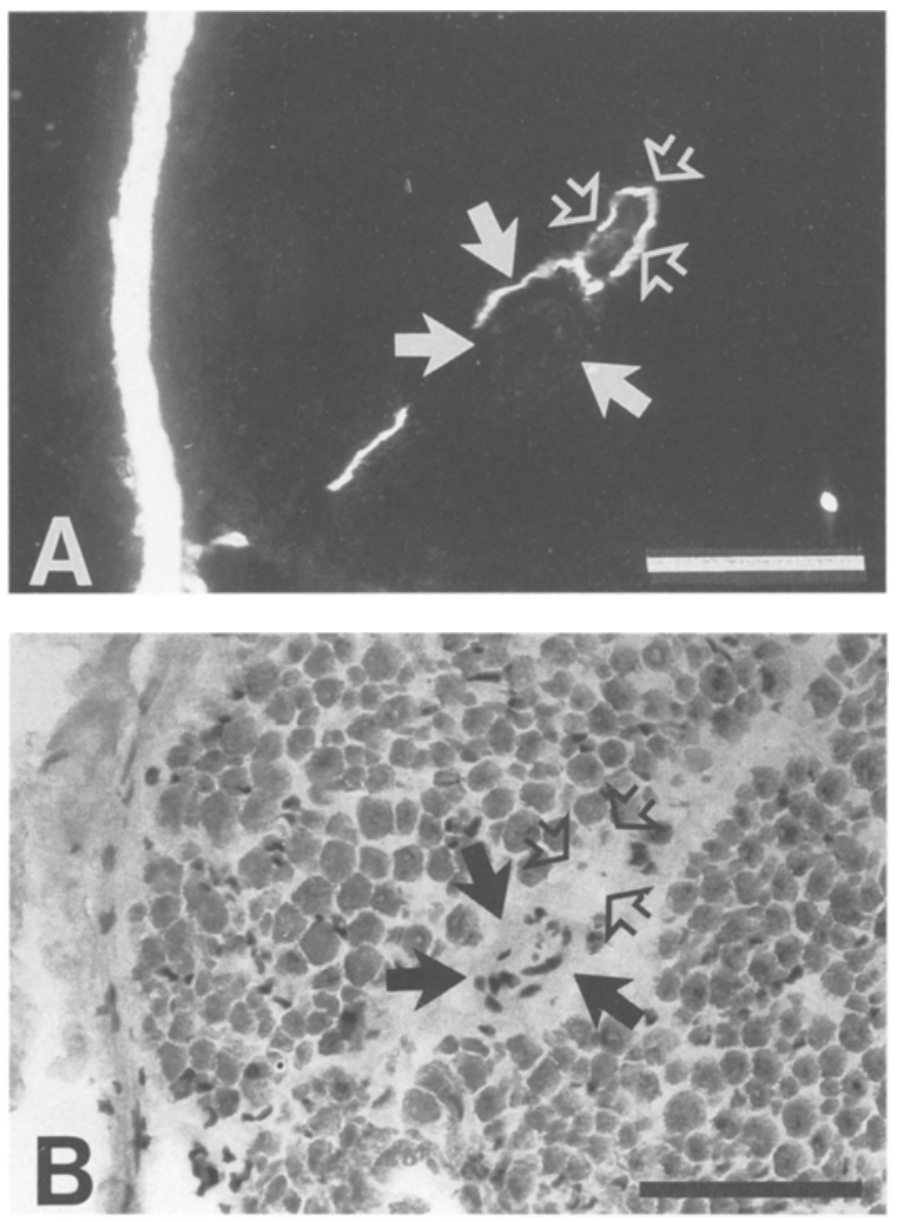

Fig.4A, B. A layer of GLUT 1 positive perineurial cells investing extracellular matrix. A Indirect immunofluorescence staining of adult human sciatic nerve for GLUT 1 . An area apparently containing only extracellular matrix is surrounded by a GLUT 1 positive sheath (open arrows). An endoneurial blood vessel is indicated with arrows. B Haematoxylin-eosin staining demonstrating the histology of the corresponding field shown in $\mathbf{A}$. $\mathbf{A}$ and $\mathbf{B}$ represent serial sections of the same sample. Bar, $100 \mu \mathrm{m}$ lial cells by morphological criteria. However, the presence of capillaries in the perineurium at 17 weeks may suggest that the GLUT 1 positive cells within the perineurium at 14 weeks represent endothelial cells. GLUT 1 epitopes were not observed in the endoneurium at this stage (Fig. 5A).

At 17 weeks of age numerous GLUT 1 positive blood vessels were seen in the endoneurium (Fig. 5B), around the perineurial tube, and between the perineurial cell layers. Both endo- and epineurial capillaries stained positively for GLUT 1 in the nerves of 17-, 19-, 22-, 26-, and 35week-old fetuses (Fig.5).

\section{Discussion}

Northern blot analysis of total RNA extracted from adult human sciatic nerve revealed a characteristic $2.8-\mathrm{kb}$ GLUT 1 mRNA transcript [3] after a relatively short $(18 \mathrm{~h})$ exposure. When samples isolated from adult or fetal (19-week-old) nerves were analysed for GLUT 3 mRNA, typical transcripts of $4.1 \mathrm{~kb}$ and $2.7 \mathrm{~kb}$ in size were visualized after a markedly longer exposure. Thus, both GLUT 1 and GLUT 3 genes are constitutively expressed in human nerve; however, GLUT 1 seems to be expressed at a markedly higher level than GLUT 3.

GLUT 1 proteins extracted from adult human and mature rat sciatic nerves had clearly different mobilities in SDS-PAGE. The migration position of the rat GLUT 1 corresponded to $55-60 \mathrm{kDa}$, as estimated by the protein markers with known molecular masses, which is in agreement with previous observations [11]. GLUT 1 of human sciatic nerve migrated more slowly and, in contrast to rat GLUT 1, consisted of two components with slightly different mobilities. None of these bands was observed when the GLUT 1 antibody was preabsorbed with a synthetic peptide identical to that originally used for immunization. Consequently, the bands are likely to represent GLUT 1 protein. The differences in the mobilities of GLUT 1 proteins extracted from human and rat nerves in SDS-PAGE may be due to different sizes of the proteins, or conformational differences. In the Western blot films an additional, high molecular mass band was observed in the samples ex- 

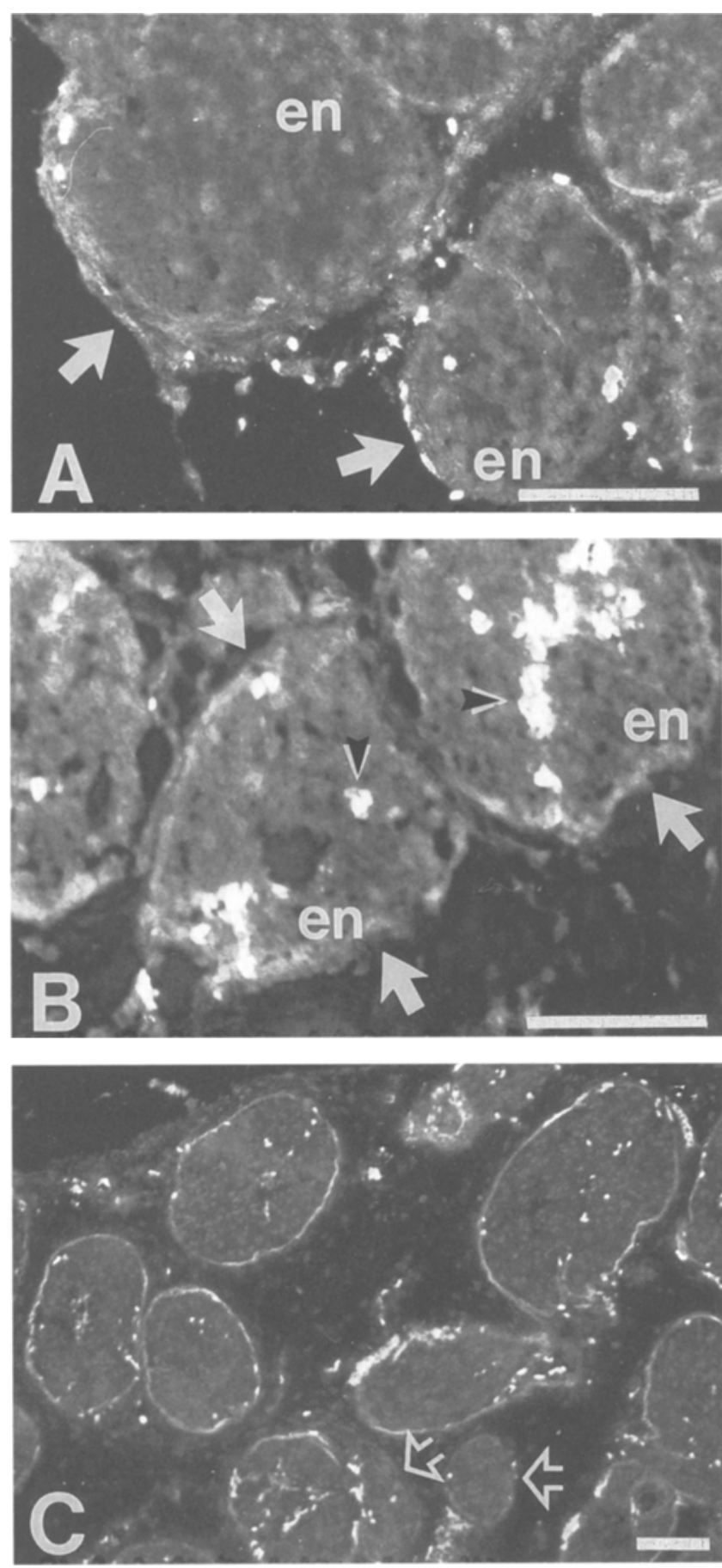

Fig.5A-F. Localization of GLUT 1 epitopes in developing human sciatic nerve. A 14 weeks: Endoneurium (en) is entirely negative for GLUT 1. Single GLUT 1 positive cells, possibly representing endothelial cells (see Results), are seen within the perineurium (large arrows). B 17 weeks: Perineurium (large arrows) is mostly negative for GLUT 1. Numerous GLUT 1 positive blood vessels are present in the endoneurium (black arrowheads). C 19 weeks: Perineuria of most fascicles show a continuous staining for GLUT 1, whereas some fascicles (open arrows) are devoid of perineurial cell-associated

tracted from human nerve. The band was not abolished by preabsorption of the GLUT 1 antiserum with the peptide used as immunogen, and was thus regarded as non-specific. It should be noted however, that preabsorption of
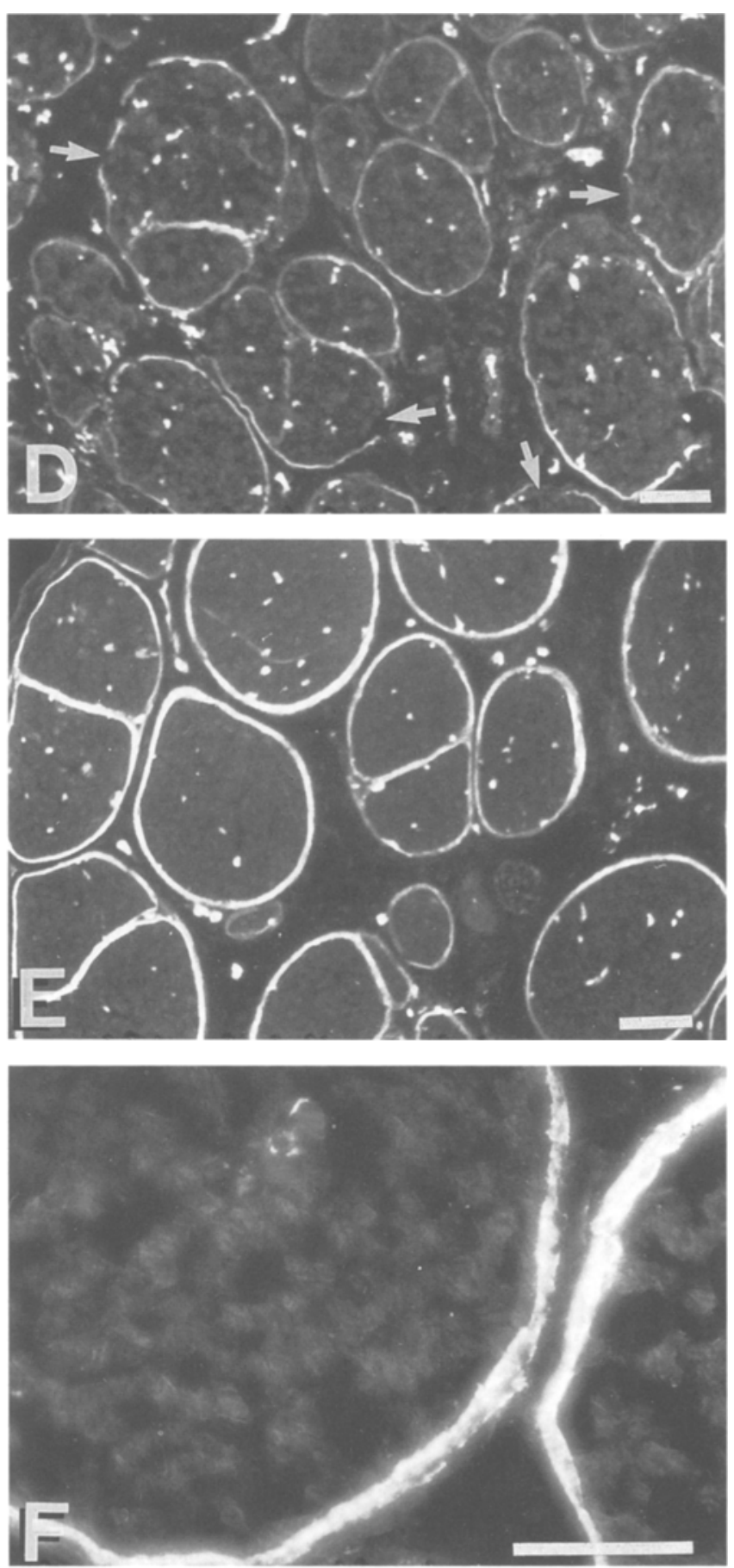

staining. D 22 weeks: Each fascicle is surrounded by a GLUT 1 positive perineurium, but the staining pattern is occasionally discontinuous (small arrows). E 26 weeks: Perineuria show a prominent and continuous staining resembling that seen in adult nerves. F 35 weeks: The perineurium is intensely stained with antibodies to GLUT 1. Note that both the endoneurial and epineurial blood vessels are positive for GLUT 1 in samples from 17-, 19-, 22- and 26-week-old fetuses. en $=$ endoneurium. Bars, A-E, $100 \mu \mathrm{m} ; \mathrm{F}, 50 \mu \mathrm{m}$

the antiserum prior to immunostainings completely eliminated the specific staining observed in the nerve tissue samples. Thus, with careful controls, the non-specific reactivity of the GLUT 1 antiserum with an unknown mole- 
cule with high molecular mass does not confound the interpretation of immunohistochemical studies of nerve tissue.

Immunostaining of adult sciatic nerve revealed that the GLUT 1 transporter concentrated in the cell layers of the perineurium. GLUT 1 positive extensions of perineurium often surrounded endoneurial blood vessels. Previously, Gerhart et al. [12] have shown that GLUT 1 negative arterioles are occasionally invested by cellular GLUT 1 positive sleeves in canine nerves and suggested that these sleeves originate from the perineurium. However, the continuity of these structures with the perineurium has not been demonstrated previously. Our present studies showed that the cellular tube originating from the perineurium and investing a blood vessel was often connected to the perineurium by a cellular sheet. On rare occasions, GLUT 1 positive perineurial sheets extended into the endoneurium and surrounded bundles of collagen fibrils. The perineurial extensions investing endoneurial blood vessels and connective tissue may have an anchoring function, stabilizing the connection between the perineurial tube and its endoneurial contents.

Surprisingly, the endothelial cells of endoneurial capillaries were only rarely positive for GLUT 1 in the adult human nerves. Previous studies on rat and dog have demonstrated that not only the perineurium, but also the endoneurial capillaries contain a high concentration of GLUT 1 epitopes in these species $[9,11,12]$. The perineurium and the endoneurial capillaries form the physical basis for the blood-nerve barrier. It has been suggested that a high density of glucose transporters is a biochemical concomitant of barrier properties in adult tissues, and that the most intense immunostaining for GLUT 1 is associated with cells bearing occluding junctions [19-21]. The lack of immunoreaction for GLUT 1 in most endoneurial capillaries of adult human nerves suggests that the expression of GLUT 1 protein in the endoneurial capillaries of human peripheral nerve may not be strictly linked with barrier function. This is in contrast to findings on nerves of rat and $\operatorname{dog}[9,11,12]$. Whether a GLUT isoform distinct from GLUT 1, possibly a yet unknown isoform, is expressed by the endoneurial capillaries of human peripheral nerve remains to be elucidated. Interestingly, Northern transfer analysis demonstrated that GLUT 3 is expressed in human sciatic nerve at the mRNA level, whereas GLUT 3 protein or mRNA have not been observed in rat sciatic nerve $[9,22]$. Thus, GLUT 3 might be a candidate for the glucose transporter of endoneurial capillaries in mature human nerves.

Perineurial cells seem to be especially sensitive to diabetic conditions with regard to changes of the extracellular matrix: the basement membranes of perineurial cells are markedly thickened in diabetes [23], and deposits of microfibrils are present in the vicinity of perineurial cells in diabetic nerves ( $P$. Muona, unpublished data). The abundance of GLUT 1 molecules on both sides of the perineurial cells suggests that transport of glucose within these cells may be exceptionally high, and thus the insulinindependent perineurial cells may be exposed to large amounts of intracellular glucose under hyperglycaemic conditions. In vitro, perineurial cells appear to partially protect themselves against high ambient glucose concentrations by down-regulating glucose uptake and the amount of glucose transporters [9]. Whether this occurs also in vivo remains to be elucidated.

In fetal sciatic nerves the distribution of GLUT 1 was strikingly different from that observed in the adult nerves, and underwent major changes during development between 14 and 35 weeks of gestational age. The positive staining of the perineurium increased gradually between weeks 14 and 35 of gestation. Interestingly, the increase of GLUT 1 expression in the perineurium was concomitant with the synthesis of structural basement membranes by perineurial cells: at 17 weeks, only short fragments of basement membrane are detected, while at 26 weeks, a large proportion of the cell surface is covered with basement membrane (S. Jaakkola, unpublished data). Together these two properties, expression of GLUT 1 and presence of basement membranes, may relate to the maturation of the perineurium into the adult-like, selectively permeable perineurial barrier. This maturation seems to be especially rapid at the age of 22-26 weeks of human development. Recently, confinement of GLUT 1 expression to the endothelium of cerebral capillaries in developing rat brain has been demonstrated to be concomitant with the maturation of the blood-brain barrier, the latter assessed by perfusion experiments and detection of a tight junction-specific protein [24].

In contrast to adult human sciatic nerves where only a few endoneurial capillaries showed positive immunoreaction for GLUT 1, both endo- and epineurial capillaries of developing human nerves between 17 and 35 weeks of gestation stained positively for GLUT 1 . This suggests that GLUT 1 may have a specific role, possibly unrelated to barrier function, in the vasculature of developing human nerve. Previous studies have shown that several tissues, such as rat liver and heart muscle, express GLUT 1 at a high level during the fetal development, whereas the expression is effectively down-regulated in the adult tissues $[14,15]$. This has been suggested to indicate a specific, though yet unknown, role for GLUT 1 in the developing tissues $[14,15]$. Demonstration of GLUT 1 positive blood vessels in the endoneurium at the age of 17 weeks of gestation but not at 14 weeks is in agreement with a previous electron microscopic study detecting endoneurial blood vessels in human sural nerve at 18 weeks but not at 16 weeks of gestation [25].

In summary, the gradual increase of staining for GLUT 1 in the perineurium during the fetal development suggests that the expression of GLUT 1 increases concomitant with the maturation of barrier properties of the perineurium $[11,26]$. On the other hand, the abundant GLUT 1 in the vasculature of developing nerves may have a specific role in the proliferating endothelial cells, since the endothelial cells of endoneurial capillaries in adult human nerves only occasionally show a detectable level of GLUT.1.

Acknowledgements. Dr. B. Thorens, Whitehead Institute for Biomedical Research (Cambridge, Mass., USA), is gratefully acknowledged for providing the GLUT 1 antiserum and the human sequence specific GLUT 1 cDNA. Dr. G. I. Bell, University of Chicago (Chicago, Ill., USA), kindly provided the human sequence specific 
GLUT 3 cDNA. Mrs. L. Peltonen provided excellent technical assistance. This work was financially supported by the Finnish Academy, the Jusélius Foundation, Nordisk Insulin Foundation Committee, and Kyllikki and Uolevi Lehikoinen Foundation.

\section{References}

1. Bell GI, Kayano T, Buse JB et al. (1990) Molecular biology of mammalian glucose transporters. Diabetes Care 13: 198-208

2. Thorens B, Charron MJ, Lodish HF (1990) Molecular physiology of glucose transporters. Diabetes Care 13: 209-218

3. Mueckler M, Caruso C, Baldwin SA et al. (1985) Sequence and structure of a human glucose transporter. Science 229: $941-945$

4. Kayano T, Fukumoto H, Eddy RL et al. (1988) Evidence for a family of human glucose transporter-like proteins. Sequence and gene localization of a protein expressed in fetal skeletal muscle and other tissues. J Biol Chem 263: 15245-15248

5. Fukumoto H, Seino S, Imura H et al. (1988) Sequence, tissue distribution, and chromosomal localization of mRNA encoding a human glucose transporter-like protein. Proc Natl Acad Sci USA 85: $5434-5438$

6. Fukumoto H, Kayano T, Buse JB et al. (1989) Cloning and characterization of the major insulin-responsive glucose transporter expressed in human skeletal muscle and in other insulin-responsive tissues. J Biol Chem 264: 7776-7779

7. Kayano T, Burant CF, Fukumoto H et al. (1990) Human facilitative glucose transporters. Isolation, functional characterization, and gene localization of cDNAs encoding an isoform (GLUT 5) expressed in small intestine, kidney, muscle, and adipose tissue and an unusual glucose transporter pseudogene-like sequence (GLUT 6). J Biol Chem 265: 13276-13282

8. Walker PS, Donovan JA, Van Ness BG, Fellows RE, Pessin JE (1988) Glucose-dependent regulation of glucose transport activity, protein, and mRNA in primary cultures of rat brain glial cells. J Biol Chem 263: 15594-15601

9. Muona P, Sollberg S, Peltonen J, Uitto J (1992) Glucose transporters of rat peripheral nerve: differential expression of GLUT-1 gene by Schwann cells and perineurial cells in vivo and in vitro. Diabetes (in press)

10. Greene DA, Winegrad AI (1979) In vitro studies of the substrates for energy production and the effects of insulin on glucose utilization in the neural components of peripheral nerve. Diabetes 28: 878-887

11. Froehner SC, Davies A, Baldwin SA, Lienhard GE (1988) The blood-nerve barrier is rich in glucose transporter. J Neurocytol 17: $173-178$

12. Gerhart DZ, Drewes LR (1990) Glucose transporters at the blood-nerve barrier are associated with perineurial cells and endoneurial microvessels. Brain Res 508: 46-50

13. Thomas PK, Olsson Y (1984) Microscopic anatomy and function of the connective tissue components of peripheral nerve. In: Dyck VJ, Thomas PK, Lambert EH, Bunge RP (eds) Peripheral neuropathy. WB Saunders, Philadelphia, pp 97-120
14. Asano T, Shibasaki Y, Kasuga M et al. (1988) Cloning of a rabbit brain glucose transporter CDNA and alteration of glucose transporter mRNA during tissue development. Biochem Biophys Res Commun 154: 1204-1211

15. Werner H, Adamo M, Lowe WL Jr, Roberts CT Jr, LeRoith D (1989) Developmental regulation of rat brain/Hep G2 glucose transporter gene expression. Mol Endocrinol 3: 273-279

16. Peterson GL (1983) Determination of total protein. Methods Enzymol 91: 95-119

17. Thorens B, Flier JS, Lodish HF, Kahn BB (1990) Differential regulation of two glucose transporters in rat liver by fasting and refeeding and by diabetes and insulin treatment. Diabetes 39 : $712-719$

18. Chomczynski P, Sacchi N (1987) Single-step method of RNA isolation by acid guanidium thiocyanate-phenol-chloroform extraction. Anal Biochem 162: 157-159

19. Harik SI, Kalaria RN, Andersson L, Lundahl P, Perry G (1990) Immunocytochemical localization of the erythroid glucose transporter: abundance in tissues with barrier functions. J Neurosci 10: $3862-3872$

20. Harik SI, Kalaria RN, Whitney PM et al. (1990) Glucose transporters are abundant in cells with "occluding" junctions at the blood-eye barriers. Proc Natl Acad Sci USA 87: 4261-4264

21. Takata K, Kasahara T, Kasahara M, Ezaki O, Hirano H (1990) Erythrocyte/HepG2-type glucose transporter is concentrated in cells of blood-tissue barriers. Biochem Biophys Res Commun 173: $67-73$

22. Maher F, Vannucci S, Takeda J, Simpson IA (1992) Expression of mouse-GLUT 3 and human-GLUT 3 glucose transporter proteins in brain. Biochem Biophys Res Commun 182: 703-711

23. Johnson PC, Brendel K, Meezan E (1981) Human diabetic perineurial cell basement membrane thickening. Lab Invest 44: 265 270

24. Dermietzel R, Krause D, Kremer M, Wang C, Stevenson B (1992) Pattern of glucose transporter (GLUT 1) expression in embryonic brains is related to maturation of blood-brain barrier tightness. Developmental Dynamics 193: 152-163

25. Ochoa J (1971) The sural nerve of the human foetus: electron microscope observations and counts of axons. J Anat 108: $231-245$

26. Burkel WE (1967) The histological fine structure of perineurium. Anat Rec 158: $177-190$

Received: 24 July 1992 and in revised form: 1 October 1992

Dr. J. Peltonen

Department of Medical Biochemistry

University of Turku

Kiinamyllynkatu 10

SF-20520 Turku

Finland 